\title{
Effects of Mn Substitution on Magnetic and Electronic Properties of $\beta$-SiC Semiconductor
}

\author{
M. Izadifard \\ Physics department, Shahrood University of Technology \\ Shahrood, P.O.BoX: 3619995161, Iran \\ Tel: 98-273-339-5270Ｅ-mail: mizadifard@yahoo.com \\ M. E. Ghazi (Corresponding author) \\ Physics department, Shahrood University of Technology \\ Shahrood, P.O.BoX: 3619995161, Iran \\ Tel: 98-273-339-5270_E-mail: ebrahim_ghazi@yahoo.com \\ S. Hosaini \\ Physics department, Shahrood University of Technology \\ Shahrood, P.O.BoX: 3619995161, Iran \\ Tel: 98-273-339-5270_E-mail: smr_hoseini80@yahoo.com
}

\begin{abstract}
Wide band gap semiconductors doped by transition metals are attracting much attention in part because of possible 'spintronics' applications. Using pseudo-potential plane-wave calculations and density functional theory (DFT), we studied effects of doping Mn of various concentrations on the cubic silicon carbide structure $(\beta-\mathrm{SiC})$. Band structures and density of states (DOSs) were calculated for $\beta-\mathrm{SiC}$ and $\mathrm{Si}_{1-\mathrm{x}} \mathrm{Mn}_{\mathrm{x}} \mathrm{C}$ (with $\mathrm{x}=0.0313,0.0625$, and 0.25). Analyses of the DOSs revealed that the diluted ferromagnetic semiconductor $\mathrm{Si}_{1-\mathrm{x}} \mathrm{Mn}_{\mathrm{x}} \mathrm{C}$, i.e. $\mathrm{SiC}$ with Mn substituted for $\mathrm{Si}$, should be a half-metal. Our results show spin polarization at Fermi energy $\left(\mathrm{E}_{\mathrm{f}}\right)$, and a stable ferromagnetic state for $\mathrm{x}=0.0625$.
\end{abstract}

Keywords: Diluted magnetic semiconductors, Spintronics, Semimagnetic semiconductors, Pseudo-potential plane-wave, Spin polarization

\section{Introduction}

Diluted magnetic semiconductors (DMSs), also referred to as semimagnetic semiconductors, in which magnetic elements are substituted for a few percent of host elements, have been intensively studied due to the potential device applications that utilize both information processing and data storage within one material system (Ohno $\mathrm{H}$. 1998, Wolf S. A. et al., 2001). The devices using DMS compounds work on the basis of spin-based electronics, also known as spintronics. It is widely believed that the ferromagnetism in such materials is carrier-induced, with holes donated by $\mathrm{Mn}$ ions mediating a ferromagnetic interaction between the randomly localized Mn spins (Sarma S. Das et al., 2003, Ohno H. et al., 1996). Coupling of carrier spins and localized moments (Mn impurities) gives rise to the unique magnetic and transport properties in DMSs. The origin of this phenomenon is still a subject of intense experimental and theoretical efforts worldwide. Most of the works done to date have widely focused on III-V DMS materials such as (Ga,Mn)As, (In,Mn)As and (Ga,Mn)N with Mn concentrations of about 5 atomic percent, which have demonstrated Curie temperatures limited to $150 \mathrm{~K}$ (Ku K. C. et al., 2003). Theoretical calculations predict that semiconductors with small spin-orbit coupling and/or consisting of light elements such as p-type wide band gap materials will have Curie temperatures $\left(\mathrm{T}_{\mathrm{C}}\right)$ above room temperature, as needed to realize functional devices (Dietl T. et al., 2000). For a successful synthesis of high- $\mathrm{T}_{\mathrm{C}}$ ferromagnetic DMSs, it is necessary to understand the origin of ferromagnetism in DMSs. Recently, attempts have been made to fabricate SiC-based DMS compounds by implanting Mn or Fe ions in high doses (Theodoropoulou N. et al., 2001). Some major advantages of $\mathrm{SiC}$ over the other wide band gap semiconductors are (i) having available large-sized substrates; (ii) developed single crystal epitaxial growth, (iii) controlled doping possibilities, and (iv) 
existence of several polytypes with different band gaps (Harris C. I. et al., 2001, Marsi P. 2002). These advantages make $\mathrm{SiC}$ a good candidate for both the spintronics and optoelectronics applications. The main building block of all polytypes of $\mathrm{SiC}$ is a tetrahedron consisting of a carbon atom bonded to four silicon atoms, and vice versa (Ching W. Y. et al., 2006, Matos M. et al., 2002).

In this work, we used ultrasoft pseudo-potential plane-wave (USPP) calculations (Vanderbilt D.1990) based on the DFT (Kohn W. et al., 1965) to study the effects of doping Mn of various concentrations on the electronic and magnetic properties of $\beta-\mathrm{SiC}$, which has a zinc blende structure.

\section{Calculation method}

In this work, we used the Plane Wave Self Consistent Field, PWscf code, which is in the framework of DFT and is based on the pseudo-potential calculation method. This code is a useful tool for calculating DOSs and band structures of materials. Calculations were first carried out on undoped $\beta$-SiC structures including two-atomic unit cell and fcc bravais lattice, and then repeated for Mn-doped samples. The ultrasoft pseudo-potentials with identical approximation for each atom of $\mathrm{Si}, \mathrm{C}$, and $\mathrm{Mn}$ were chosen, and then in order to start the calculations, the lattice parameters, k-points in the first brillouin zone, and cut-off energy for electronic wave function were optimized. Self and non-self consistent calculations were carried out using these optimized values. Total energies for the $3.13 \%, 6.25 \%$, and $25 \% \mathrm{Mn}$ concentrations were calculated.

\section{Results and discussion}

Starting with the undoped sample, a unit cell with two-atomic basis and fcc Bravais lattice was chosen. The optimized values for cut-off energy and lattice parameter were 28 Ry and 8.28 a.u., respectively. The optimized lattice parameter is in agreement with the reported experimental value, which is 8.23 a.u. (Madelung O. et al., 1982, Gubanov V. A. et al., 2001). Figure 1 shows the calculated band structure for $\beta$-SiC along the high symmetry direction in the first Brillouin zone, which is in accordance with the other results (Ohno H. 1998, Ching W. Y. et al., 2006, Matos M.2002). As it could be seen in the figure, this semiconductor has an indirect band gap. Maximum of the valence band is at $\mathrm{k}$-point of $\Gamma(\mathrm{k}=0,0,0)$, and minimum of the conduction band is at $X(k=2 \pi / a, 0,0)$. The value for this indirect band gap, $E_{i}$, is $1.37 \mathrm{eV}$, and the Fermi energy, $\mathrm{E}_{\mathrm{f}}$, the highest occupied level, is $9.27 \mathrm{eV}$. The value obtained for the band gap is in the range of reported values (Ching W. Y. et al., 2006).

The calculated parameters of interest are tabulated in Table 1 . The calculated total density of states (TDOSs) and partial density of states (PDOSs) for $3 \mathrm{~s}$ and $3 p$ orbitals of Si and for $2 \mathrm{~s}$ and $2 \mathrm{p}$ orbitals of $\mathrm{C}$ are shown in Figures 2, 3(a), and 3(b). As shown in Figure 2, valence band region for $\beta$-SiC is separated by a small gap. The lower part comes from bonding between $\mathrm{C}: 2 \mathrm{~s}$ and $\mathrm{Si}: 3 \mathrm{~s}$ states, whereas the upper part comes from bonding of $\mathrm{Si}: 3 \mathrm{p}$ and $\mathrm{C}: 2 \mathrm{p}$ states.

In Figures 2 and 3, the zero energy level was chosen at maximum of the valence band. Since the C:2p and Si:3p stares are mostly distributed around the valence band edge, the $\mathrm{p}$ orbitals are likely to occupy states near the Fermi energy. The states which are distributed at the edge of the conduction band are mixture of all the Si:3p,3s and C:2p,2s states. Figure 3(b) shows that DOSs related to Si atoms are higher than those for C atoms.

Manganese doping causes replacement of atoms from their equilibrium positions and change of lattice parameters due to interactions between atoms. Thus we had to minimize the interactions and interatomic forces by use of relaxation calculations. The self-consistent calculations were carried out to achieve the interatomic forces of the order of $0.0136 \mathrm{eV} / \mathrm{a} . \mathrm{u}$ (minimum value $0.001 \mathrm{Ry} / \mathrm{a} . \mathrm{u}$ ). To study the effects of Mn doping, we considered three atoms ( $\mathrm{Si}, \mathrm{C}$, and $\mathrm{Mn}$ ) in our calculations. We had to substitute one of the $\mathrm{Si}$ or $\mathrm{C}$ atoms for $\mathrm{Mn}$. We chose to replace Si with Mn because of its higher ferromagnetic state stability (Kim Y. S. et al., 2004). By selecting 8 unit cells with cubic Bravias lattice including one Mn atom, concentration of Mn reached $25 \%$. After the optimization process, cut-off energy of 28 Ry and lattice parameter of 8.28 a.u. were obtained. In a similar way, we chose a $2 \times 2 \times 2$ supercell with 64 atoms. By replacing one Si atom for a Mn atom, concentration of Mn reached 3.13\%. By replacing two $\mathrm{Si}$ atoms for two $\mathrm{Mn}$ atoms, concentration of $\mathrm{Mn}$ reached $6.25 \%$. The calculated TDOSs for the spin-up and spin-down states with different Mn concentrations are shown in Figures 4(a), 4(b), and 4(c). Zero energy level in these figures coincides the Fermi energy.

As shown in these figures, TDOSs near Fermi energy have only the spin-up state, which means that the electron spins are completely polarized. Thus spin-up states have metallic behavior and spin-down states have semiconductor behavior. According to the figures, maximum spin-up states at Fermi energy occurs for the Mn concentration of $6.25 \%$. The $3 \mathrm{~d}$ local DOSs for Mn atoms of different concentrations are shown in Figures 5 and 6. Since $\mathrm{d}$ electrons of $\mathrm{Mn}$ atoms occupy states near the Fermi level, the electronic structure of $\beta-\mathrm{SiC}: \mathrm{Mn}$ 
depends on the characteristics of the partially filled d-orbitals. These $\mathrm{d}$ states can be coupled with $\mathrm{sp}^{3}$ dangling bonds of Si, resulting in a half-metallic band structure in which the Fermi level lies in the energy gap of the minority states and thus the electrons are fully spin-polarized at $\mathrm{E}_{\mathrm{f}}(\mathrm{Kim} \mathrm{Y}$. S. et al., 2006). Calculated magnetic moments for the three Mn concentrations are listed in Table 2; these values are in good agreement with the reported values (Madelung O. et al., 1982). A high magnetic moment shows a stable ferromagnetic phase in $\mathrm{SiC}: \mathrm{Mn}$.

\section{Conclusion}

In this work, we used the pseudo-potential plane-wave method and the DFT to study effects of doping Mn of various concentrations on the $\beta$-SiC structure. Band structures and DOSs were calculated for pure $\beta$-SiC and $\mathrm{Si}_{1-\mathrm{x}} \mathrm{Mn}_{\mathrm{x}} \mathrm{C}$ (with $\mathrm{x}=0.0313,0.0625$, and 0.25 ). The calculated band structure for $\beta$-SiC showed that this semiconductor has an indirect band gap of $\mathrm{E}_{\mathrm{i}}$, equal to $1.37 \mathrm{eV}$.

Analyses of total DOSs of $\mathrm{Si}_{1-\mathrm{x}} \mathrm{Mn}_{\mathrm{x}} \mathrm{C}$ near Fermi energy revealed that only the spin-up state exists, which means that the electron spins are completely polarized. Thus spin-up states have metallic behavior and spin-down states have semiconductor behavior. According to the results, maximum spin-up states at Fermi energy occurs for the Mn concentration of $6.25 \%$. The calculation of $3 \mathrm{~d}$ local DOSs for Mn atoms of different concentrations indicated $\mathrm{d}$ electrons of $\mathrm{Mn}$ atoms occupy states near the Fermi level. Our calculations showed a spin polarization at $\mathrm{E}_{\mathrm{f}}$ and a stable ferromagnetic state for the Mn concentration of $6.25 \%$.

\section{References}

Ching W. Y., Nianxu Y., Rulis P., \& Ouyang L. (2006). The electronic structure and spectroscopic properties of 3C, 2H, 4H, 6H, 15R and 21R polymorphs of SiC. Materials Science and Engineering, A 422, 147-156.

Dietl T., Ohno H., Matsukura F., Cibert J., \& Ferrand D. (2000). Zener Model Description of Ferromagnetism in Zinc-Blende Magnetic Semiconductors. Science, 287, 1019.

Gubanov V. A., Boekema C., Fong C. Y. (2001). Electronic structure of cubic silicon-carbide doped by $3 d$ magnetic ions. Appllied Physics Letter, 178, 2.

Harris C. I., Savage S., Konstantinav A., \& Bakowski M. (2001). Progress towards SiC products. Applied Surface Science, 184, 393.

Kim Y. S., Kim H. \& Chung Y. C. (2004). Magmetic and half-metalic properties of Cr doped $\beta$-Sic. Key Engineering Materials, 264-268, 1237-1240.

Kim Y. S., Chung Y. C., \& Yi S. C. (2006). Electronic structure and half-metallic property of Mn-doped $\beta$-SiC diluted magnetic semiconductor. Materials Science and Engineering, B 126, 194-196.

Kohn W. \& Sham L. J. (1965). Self-Consistent Equations Including Exchange and Correlation Effects. Physics Review, 140, A1133-A1138.

Ku K. C., Potashnik S. J., Wang R. F., Chun S.H., Schiffer P., Samarth N., Seong M.J., Mascarenhas A., Johnston-Halperin E., Myers R. C., Gossard A. C., \& Awschalom D. D. (2003). Highly enhanced Curie temperature in low-temperature annealed [Ga,Mn]As epilayers. Applied Physics Letter, 82, 2302.

Madelung O., Schulz M., \& Weiss H. (1982). Landolt-Bornstein New series group III, vol.17a, springer verleg, New York.

Marsi P. (2002). Silicon carbide and silicon carbide-based structures: The physics of epitaxy. Surface Science Report., 48, 1.

Matos M. (2002). Electronic structure of several polytypes of $\mathrm{SiC}$ : a study of band dispersion from a semi-empirical approach. Physica, B 324, 15-33.

Ohno H., Shen A., Matsakura F., Oiwa A., Endo A., Katsumoto S., \& Iye Y. (1996). (Ga,Mn)As: A new diluted magnetic semiconductor based on GaAs. Applied Physics Letter, 69, 363.

Ohno H. (1998). Making Nonmagnetic Semiconductors Ferromagnetic. Science, 281, 951.

Sarma S. Das, Hwang E. H., \& Kaminski A. (2003). Temperature-dependent magnetization in diluted magnetic semiconductors. Physics Review, B 67, 155201.

Theodoropoulou N., Hebard A. F., Chu S.N.G., Overberg M. E., Abernathy C.R., Pearton S. J., Wilson R.G. \& Zavada J. M. (2001). Magnetic Properties of Fe- and Mn-Implanted SiC. Electrochemical and Solid State Letters, 4, G119. 
Vanderbilt D. (1990). Soft self-consistent pseudopotentials in a generalized eigenvalue formalism. Physics Review, B 41, 7892.

Wolf S. A., Awschalom D., Buhrman R. A., Daughton J. M.,. molnar S.Von, Roukes M. L., Chtchelkanova A.Y., \& Treger D. M. (2001). Spintronics: A Spin-Based Electronics Vision for the Future. Science, 294, 1488.

Table 1. Calculated parameters of interest for $\beta$-SiC

$\begin{array}{lll}\beta-\mathrm{SiC} & \text { Our results } & \text { Other results }\end{array}$
Direct band gap (eV)
5.56
$4.86[10]$

Indirect band gap (eV)

1.37

$1.317[16], 1.602[10]$

Width of upper valence band $(\mathrm{eV})$

8.93

$8.59[10]$

Width of lower valence band $(\mathrm{eV})$

5.65

$5.38[10]$

Width of conduction band $(\mathrm{eV})$

18.25

Table 2. Calculated total magnetic moments and spin band gaps for $\beta$-SiC

\begin{tabular}{cccccc}
\hline Mn doping (\%) & $\begin{array}{c}\mathrm{E}_{\mathrm{f}} \\
(\mathrm{eV})\end{array}$ & $\begin{array}{c}\text { Total magnetization } \\
\left(\mu_{\mathrm{B}} / \mathrm{cell}\right)\end{array}$ & $\begin{array}{c}\mathrm{E}_{\mathrm{f}}-\mathrm{E}_{\mathrm{VBM}} \\
(\mathrm{eV})\end{array}$ & $\begin{array}{c}\mathrm{E}_{\mathrm{CBM}}-\mathrm{E}_{\mathrm{f}} \\
(\mathrm{eV})\end{array}$ & $\begin{array}{c}\text { Spin down } \\
\text { band gap }(\mathrm{eV})\end{array}$ \\
\hline $3.13 \%$ & 10.84 & 3.05 & 1.2 & 0.34 & 1.54 \\
\hline $6.25 \%$ & 10.94 & 3.00 & 1.12 & 0.26 & 1.38 \\
\hline & 10.76 & 3.00 & 1.96 & 0.27 & 2.23 \\
\hline
\end{tabular}




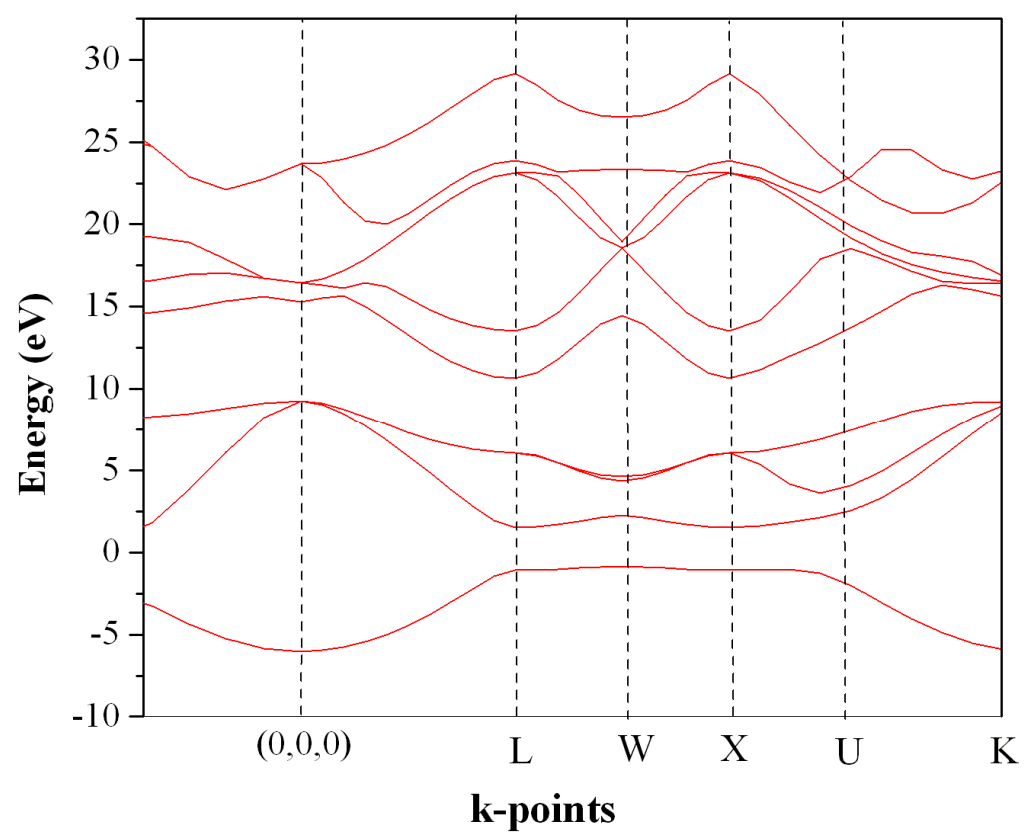

Figure 1. Calculated electronic band structure for $\beta-\mathrm{SiC}$

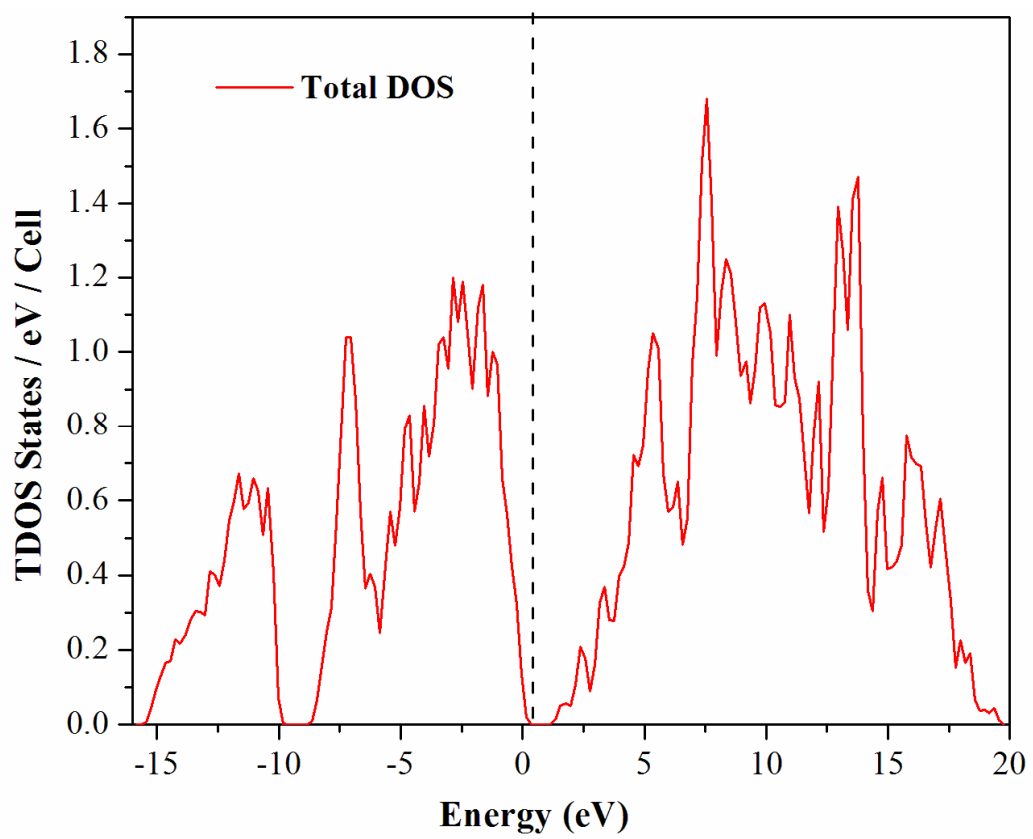

Figure 2. Calculated TDOS for $\beta$-SiC structure. The zero energy level corresponds to maximum of the valence band 

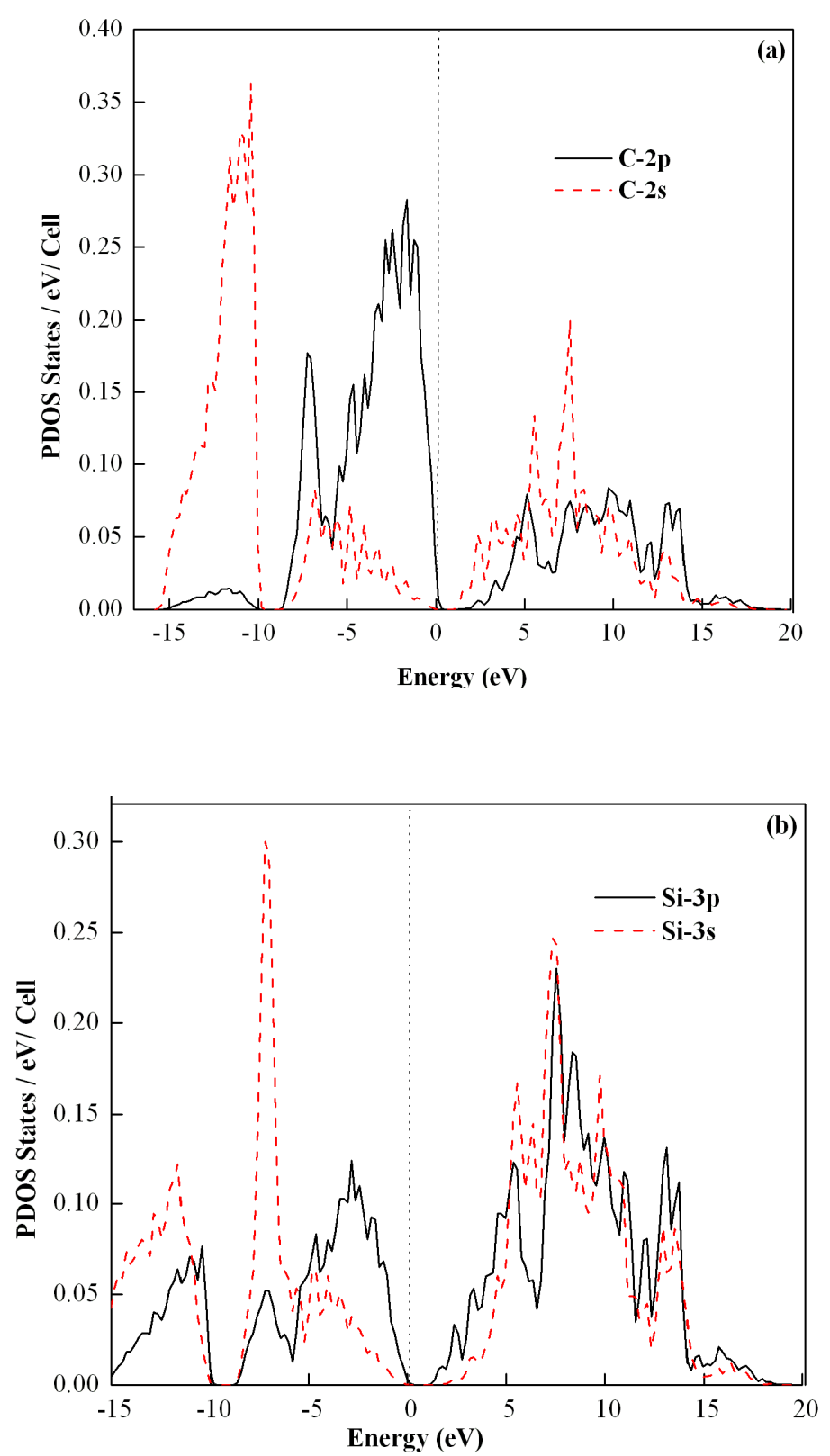

Figure 3. Calculated PDOSs for (a) C:2s and C:2p; and (b) Si:2s and Si:2p 

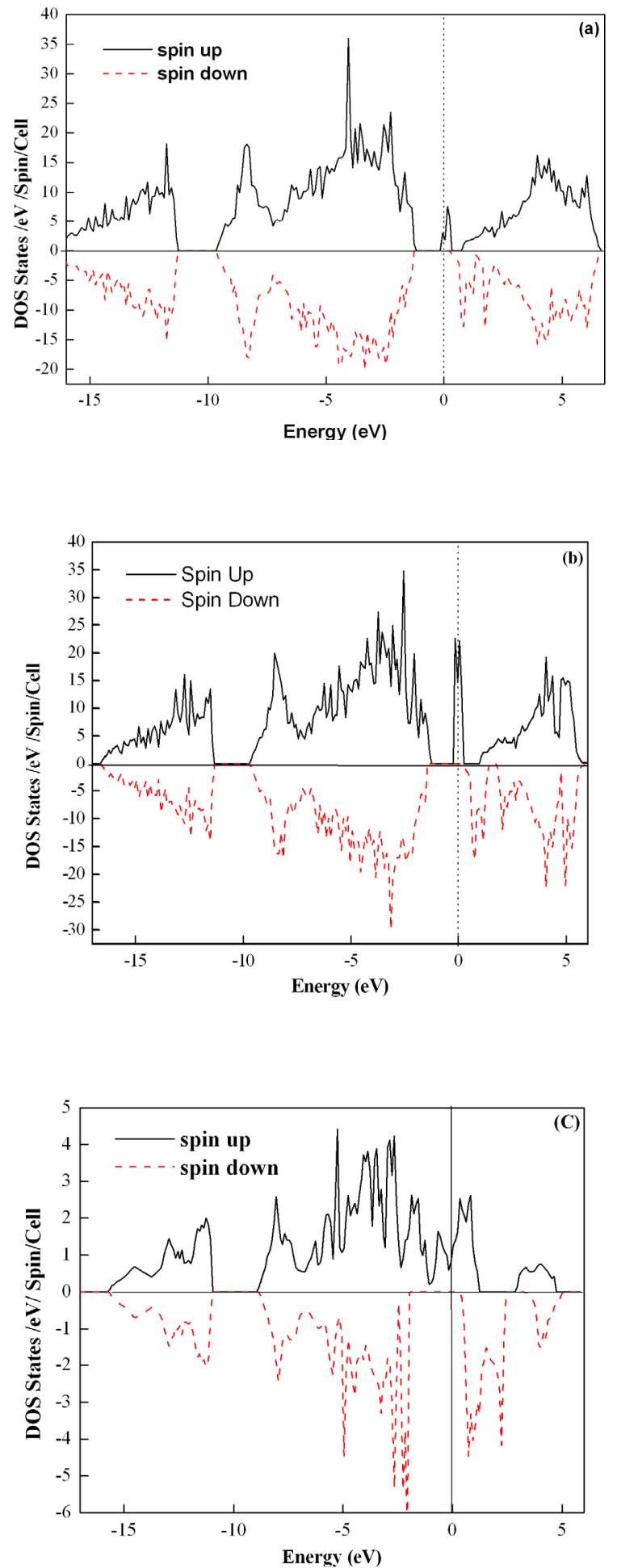

Figure 4. Calculated TDOSs for (a) 3.13\% $\mathrm{Mn}$, (b) 6.25\% $\mathrm{Mn}$, and (c) 25\% $\mathrm{Mn}$. 


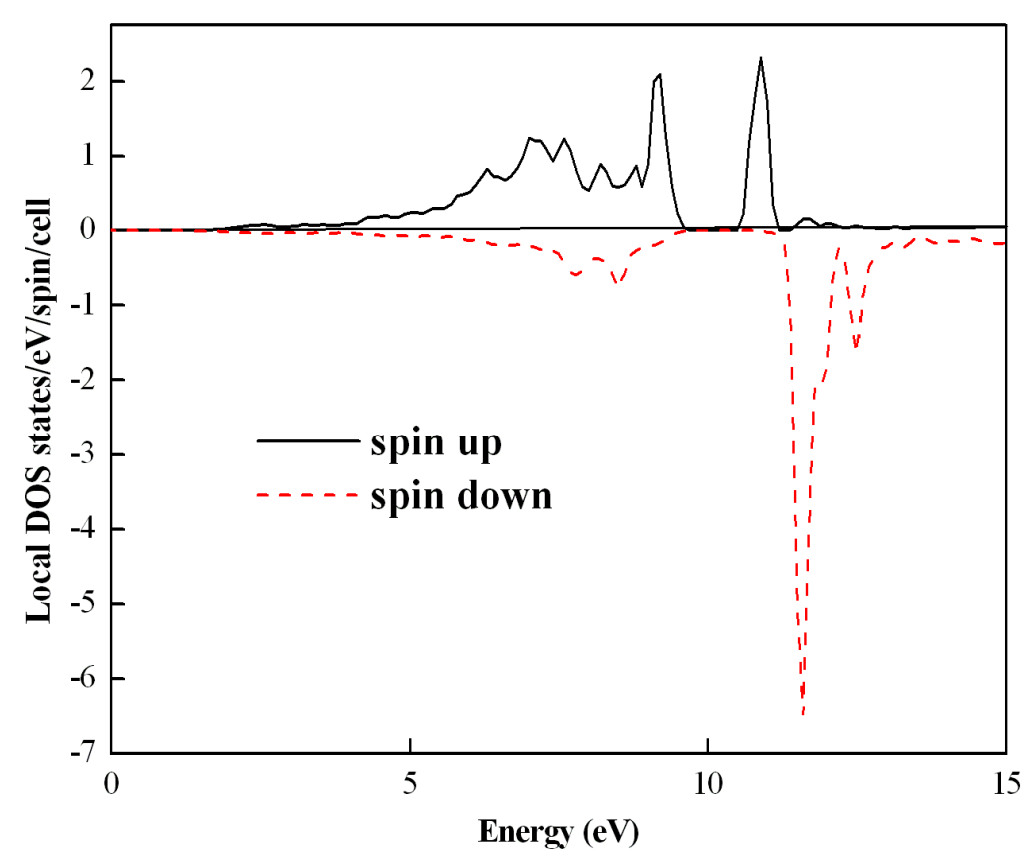

Figure 5. 3d local DOSs for Mn concentration of 3.13\%

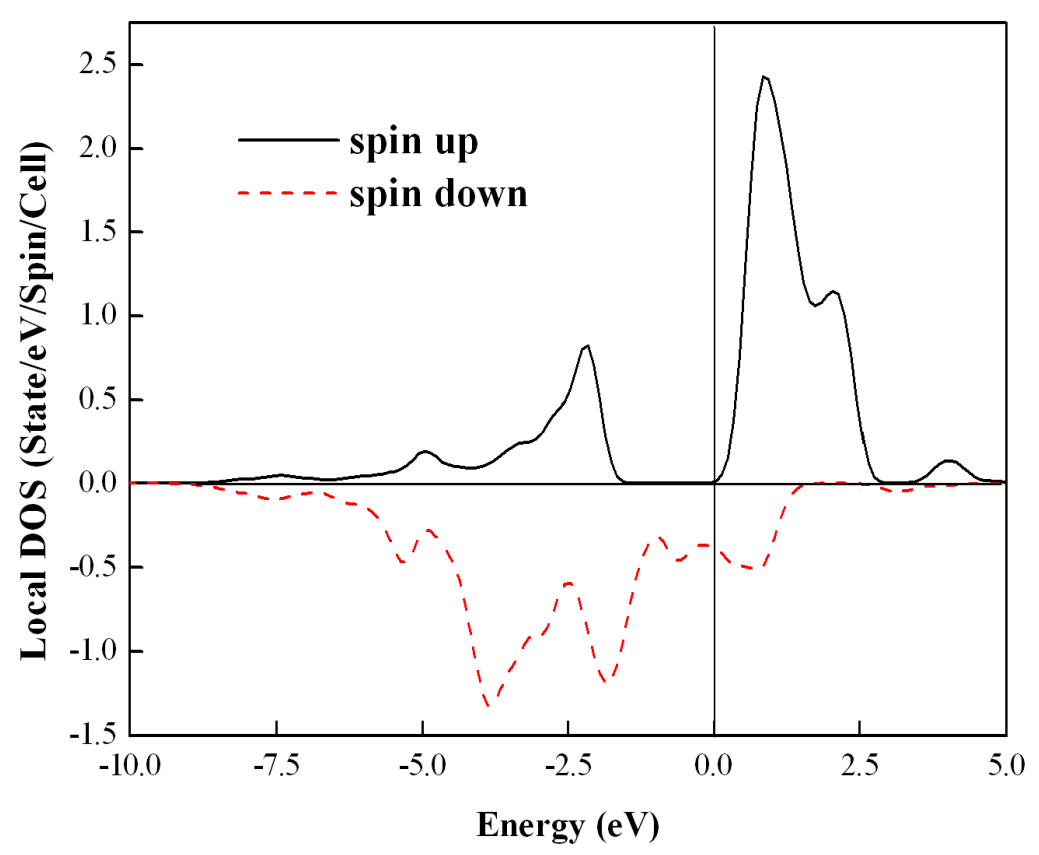

Figure 6. 3d local DOSs for Mn concentration of 25\% 